\title{
Density of States and Photoluminescence Spectra in the Dense Arrays of Epitaxial CdSe/ZnSe Quantum Dots with Gaussian Potential Profile
}

\author{
A.A. Golovatenko*, M.A. Semina, A.V. Rodina and T.V. Shubina \\ Ioffe Institute, 26 Politekhnicheskaya, St. Petersburg 194021, Russia
}

\begin{abstract}
The paper reports on the studies of excitonic level arrangement in dense arrays of CdSe/ZnSe quantum dots grown by molecular beam epitaxy. Our modeling, exploiting experimental data obtained by optical and transmission electron microscopy studies, has exhibited inconsistency between the energy and width of experimental emission band and those predicted by a straightforward model for calculation of the density of excitonic states in the dots. To reach an agreement, we assume possibility of energy transfer between the adjacent dots of different sizes and propose ways for its experimental verification.
\end{abstract}

DOI: 10.12693/APhysPolA.129.A-107

PACS/topics: 73.21.La, 78.55.Et, 71.35.--y

\section{Introduction}

Epitaxial II-VI quantum dots (QDs) formed by insertion of $1 \div 3$ CdSe monolayers (ML) within a $\mathrm{Zn}(\mathrm{S}, \mathrm{Se})$ matrix have been studied from the end of the last century [1]. The epitaxial CdSe QDs have certain advantages over the colloidal ones, namely, comparability with heterostructures of different designs and functions and the absence of blinking. The $\mathrm{CdSe} / \mathrm{ZnSe} \mathrm{QD}$ insertion was commonly considered as a disordered $\mathrm{ZnCdSe}$ quantum well with Cd-rich localization islands [2], mainly based on cross-section transmission electron microscopy (TEM) data. The $\mathrm{Cd}$ content in these islands was found to vary from $20 \%$ to $80 \%$ [3-5]. The development of molecular beam epitaxy (MBE) during the last decade has led to fabrication of efficient II-VI lasers pumped optically or by electron beam, where the array of $\mathrm{CdSe} / \mathrm{ZnSe} \mathrm{QDs}$ serves as an active region $[6,7]$. A single-photon source operating at $220 \mathrm{~K}$ exploited the CdSe/ZnSe QD inserted in a nanowire to select a single excitonic line [8]. Later, a single-photon source, operating at room temperature was obtained [9]. Further progress in these fields requires detailed information on the arrangement of exciton energy levels in QD arrays and the possibility to select limited number of QDs needed for the nanoemitters of quantum light. Here we report on TEM and optical studies of the dense arrays of the epitaxial CdSe/ZnSe QDs supported by the theoretical consideration of the excitonic energy levels in the QDs with the Gaussian potential profile. Our results show that the QDs arrays comprising the separated nanoislands demonstrate a tendency to bimodal size distribution and specific spatial arrangement. The architecture of quantum levels in subsystems is suggestive of the possibility of efficient energy transfer within the QD arrays.

*corresponding author; e-mail: sasha.pti@mail.ioffe.ru

\section{Experimental}

We investigated the dense arrays of $\mathrm{Cd}_{x} \mathrm{Zn}_{1-x}$ Se QDs with the QD density of $10^{11}-10^{12} \mathrm{~cm}^{-2}$ formed by MBE by inserting a single CdSe layer into a ZnSe matrix. For the sake of demonstrativeness, we focused on a layer with the nominal CdSe thickness of 2.1 ML. This sample was studied by TEM, photoluminescence spectroscopy (PL) and photoluminescence excitation spectroscopy (PLE). The specimen for plan-view TEM studies has to be thin to allow electrons to transmit. The procedure of its preparation includes dimple grinding from the substrate side followed by etching. As a result, specimen material is gradually thinned from periphery to center, up to the formation of an aperture there. Its top surface remains plane, as-grown. Thus, the reduction of the thickness takes place along the coordinate $x$ in plane. It allows us to trace how does the size distribution of QDs change not only in plane, but also along the growth direction. In the center region of the sample where the thickness is minimal, the mean size of QD does not exceed $4 \mathrm{~nm}$ (Fig. 1a,c). In the periphery region of the sample the sizes of QDs are distributed within the range 3-12 nm with maximum at $5 \mathrm{~nm}$ (Fig. 1b,d). In both cases QDs look like well separated objects in these plan-view images, despite their rather high density (Fig. 1). The array of QDs is inhomogeneous in both lateral and vertical directions. Actually, it consists of two subarrays with different size dispersion: large QDs, whose position corresponds to the initial insertion of CdSe and small QDs right above them. This distribution may be explained by the $\mathrm{Cd}$ segregation along the growth direction during re-growing of the CdSe QD array by the top ZnSe barrier [4]. Such architecture provides seeming broadening of the CdSe insertion revealed previously by XRD studies [10] and cross-section TEM studies [3].

The low-temperature PL spectroscopy studies of the sample revealed a narrow PL line $(\mathrm{FWHM} \approx 50 \mathrm{meV}$ ) with the peak at $2.54 \mathrm{eV}$. The width of the PL line in 

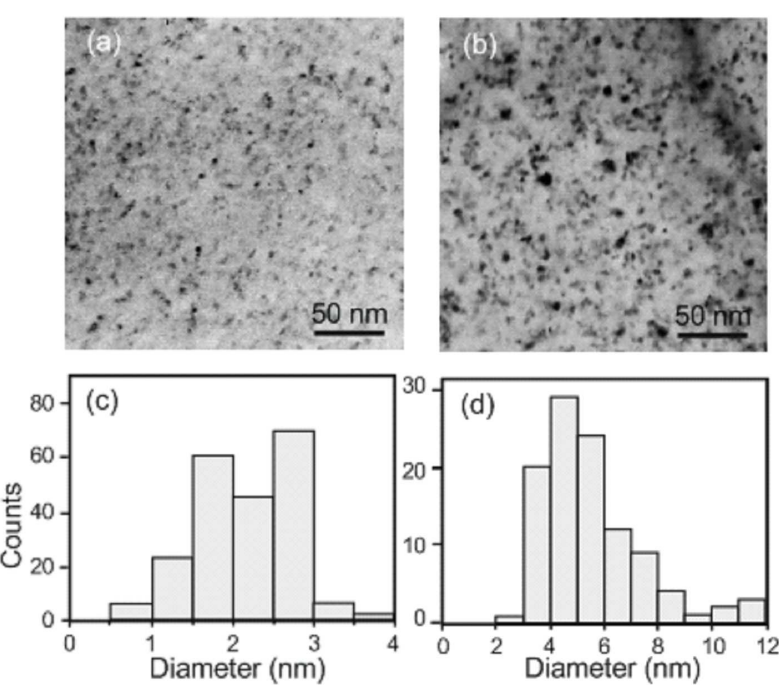

Fig. 1. Plane view TEM images $(\mathrm{a}, \mathrm{b})$ with respective histograms of QD size distribution (c,d). (a,c) top part of a CdSe insertion, where the small islands are formed mainly due to the segregation and diffusion during $\mathrm{ZnSe}$ growth. $(\mathrm{b}, \mathrm{d})$ the thick part of the insertion.

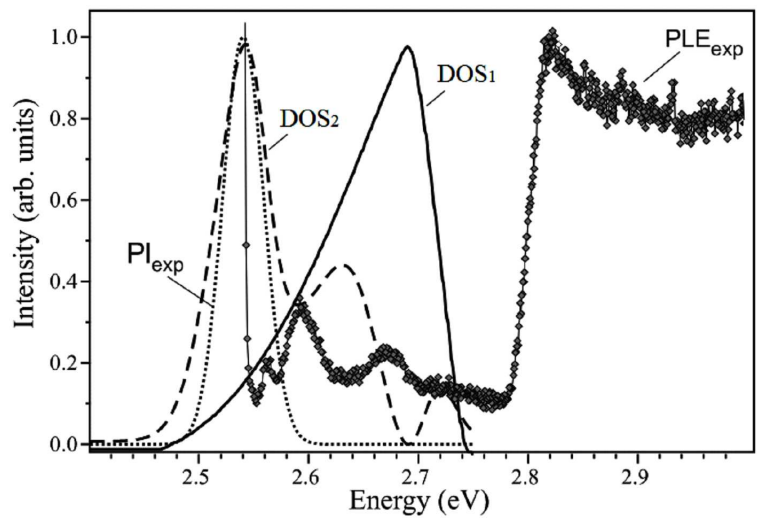

Fig. 2. Experimental PL (dotted line) and PLE (diamonds line) spectra shown together with the spectra of density of states $\mathrm{DOS}_{1}$ (solid line) and $\mathrm{DOS}_{2}$ (dashed line), calculated as described in the text. The spectra are normalized to the maximal intensity values.

the structures with $\mathrm{CdSe} / \mathrm{ZnSe} \mathrm{QDs}$ varies in the range 20-80 meV [1, 2, 11], being dependent on technological features, which determine the density and size of QDs. As a rule, these structures were treated as disordered quantum wells. However, such a consideration is not consistent with our TEM data showing distinct and wellseparated islands. Moreover, at low temperatures the recorded size distribution of the Cd-rich islands should result in a much broader PL line, regardless of whether they are real well-separated QDs or localization sites in a disordered QW. Any of them can be emitting at the low temperatures when carrier escape is suppressed. A PLE spectrum, measured with detection at the $2.52 \mathrm{eV}$, comprises three pronounced peaks, besides the excitonic peak in the ZnSe barrier (Fig. 2). The first at $2.57 \mathrm{eV}$ is narrow and can be related to LO phonon-assisted transitions.
Two other at $2.60 \mathrm{eV}$ and $2.68 \mathrm{eV}$ are smoother and have a width close to that of the PL line; their origin is under question. Such a situation impels us to search an alternative model, which could explain emerging the narrow PL line in so inhomogeneous system, as well as the observed PLE peculiarities.

\section{Modeling}

To find an explanation for contradiction between the PL width and QD size distribution we have performed modeling of the density of ground states of excitons in the QDs of different sizes belonging to this array. The density of states (DOS) can be found by replacement of the characteristic diameter of the QDs in the TEM histograms by the corresponding exciton energies. To do that we have to establish the form of confinement potential in the QDs, assuming that these epitaxial QDs are the CdZnSe ones and possess the maximal $\mathrm{Cd}$ content in the center, which is gradually decreased towards periphery $[2,3]$. Then, we suggest that the change of $\mathrm{Cd}$ content reflects the change of the alloy bandgap from $E_{\mathrm{g}}\left(\mathrm{Cd}_{x} \mathrm{Zn}_{1-x} \mathrm{Se}\right)$ to $E_{\mathrm{g}}(\mathrm{ZnSe})$ in the surrounding barriers. In this case, the spatial potential for electrons and holes can be written in the form of anisotropic Gaussian potential

$$
V_{\mathrm{G}}(r)=V_{\text {off }}\left[1-\exp \left(-\rho^{2} / a_{x}^{2}-z^{2} / a_{z}^{2}\right)\right],
$$

where $\rho$ and $z$ are the lateral and vertical distances from the center of the $\mathrm{QD}, V_{\text {off }}$ is the conduction band offset for electron or the valence band offset for hole; characteristic sizes $a_{x}$ and $a_{z}$ are of the order of the $\mathrm{QD}$ radius in the lateral and vertical directions, correspondingly. The values of the band offsets are determined as $0.25 \Delta E_{\mathrm{g}}$ for holes and $0.75 \Delta E_{\mathrm{g}}$ for electrons, where $\Delta E_{\mathrm{g}}$ is the difference between the bandgap of the ZnSe matrix and the bandgap of the $\mathrm{Cd}_{x} \mathrm{Zn}_{1-x}$ Se in the center of the QD. Following values of band gaps were used: $E_{\mathrm{g}}(\mathrm{ZnSe})=2.82 \mathrm{eV}$, $E_{\mathrm{g}}(\mathrm{CdSe})=1.74 \mathrm{eV}$ and $E_{\mathrm{g}}\left(\mathrm{Cd}_{x} \mathrm{Zn}_{1-x} \mathrm{Se}\right)=$ $(x) E_{\mathrm{g}}(\mathrm{CdSe})+(1-x) E_{\mathrm{g}}(\mathrm{ZnSe})-x(1-x) C$, where $C=0.387[12]$.

The energy of the exciton ground state in the QDs has been calculated using the effective mass approximation method in the strong confinement regime for both electron and hole. The degeneracy of the $\Gamma_{8}$ valence band was taken into account in the calculations of the ground state energy for holes. For this purpose it is convenient in the zero approximation to consider the case of spherically symmetric potential $V_{\mathrm{G}}(\mathrm{r})$ when $a_{x}=a_{z}$ [13]. In this case the ground state of hole can be described by combination of radial wave functions [14], which can be found by variational method. Solving separately the Schrödinger equations for electron and hole we find corresponding ground state energies $E_{\mathrm{e}}$ and $E_{\mathrm{h}}$. Energy of the Coulomb interaction $E_{\mathrm{C}}$ between carriers, as well as splitting between light and heavy hole energy states $E_{\Delta}$ caused by QD anisotropy, were estimated within the framework of the first order perturbation theory. The resulting exciton ground state energy reads as 


$$
E_{\mathrm{EX}}=E_{\mathrm{e}}+E_{\mathrm{h}}-E_{\mathrm{C}}-0.5 E_{\Delta} .
$$

Energy $E_{\mathrm{Ex}}$ can vary in the wide range depending on size of QD and content of $\mathrm{Cd}$ in the center of the QD. Comparing the calculated values of $E_{\mathrm{Ex}}$ with the peak position of experimental PL we find out that corresponding QDs in the studied array must have the content of Cd $x>0.6$ and the average size of $8 \mathrm{~nm}$ (Fig. 3). For further modeling of the DOS in this QD array we assume that the mean value of $\mathrm{Cd}$ concentration $x=0.8$ (Fig. 3).

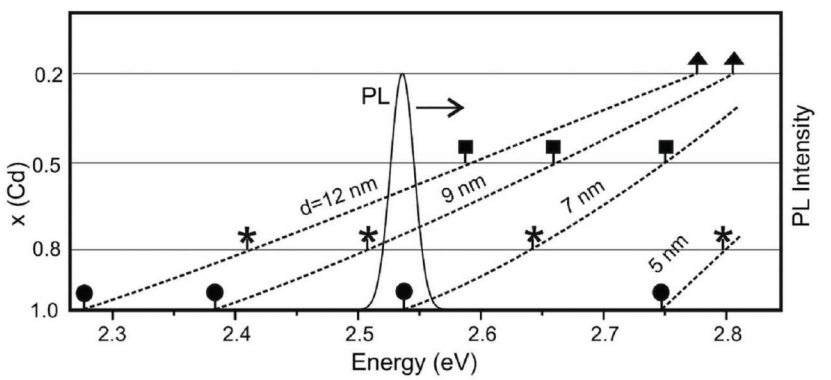

Fig. 3. Spectral dependences of the exciton ground state energy on the $\mathrm{Cd}$ content and QD characteristic diameter. A typical PL spectrum (solid line), shown for reference, was measured at low temperature $(77 \mathrm{~K})$ in a structure with 2.1 ML CdSe.

As it can be seen in Fig. 2 the width of the calculated $\mathrm{DOS}_{1}$ spectrum (solid line) is significantly larger than the width of the experimental PL spectrum (dotted line). Moreover, $\mathrm{DOS}_{1}$ spectrum has a peak at $2.7 \mathrm{eV}$, while PL peak position is $2.54 \mathrm{eV}$. At the same time, the $\mathrm{DOS}_{1}$ peak coincides with the high-energy peak in PLE spectrum (diamonds line). To explain these findings we assume that small dots, determining the maximum of the DOS $_{1}$ spectrum, do not emit by themselves but transfer the excitation to the radiating dots of larger sizes. In other words, we suggest the Förster-like mechanism of the energy transfer [15]. This mechanism is most efficient in the resonant case, when the excited excitonic states in the large QDs coincide in energy with the ground states in the small dots (Fig. 2).

The energy transfer takes place via dipole-dipole interaction between the excitonic states in the QDs of different sizes, providing that these states are overlapped. If the rate of such resonant transitions between QDs is much higher than the radiative recombination rate of excitons in the small QDs, the efficient transfer between dots can be realized. The transfer can be described by the following system of rate equations:

$$
\begin{aligned}
& \frac{\mathrm{d} N_{\mathrm{d}}(E)}{\mathrm{d} t}=-N_{\mathrm{d}}(E)\left(\Gamma+\Gamma_{\mathrm{ET}}\right) \\
& \quad+G N_{\mathrm{DOS}_{1}}(E) f_{\mathrm{d}}(E), \\
& \frac{\mathrm{d} N_{\mathrm{i}}(E)}{\mathrm{d} t}=-N_{\mathrm{i}}(E) \Gamma+G N_{\mathrm{DOS}_{1}}(E)\left[1-f_{\mathrm{d}}(E)\right],
\end{aligned}
$$

$$
\frac{\mathrm{d} N_{\mathrm{a}}(E)}{\mathrm{d} t}=-N_{\mathrm{a}}(E) \Gamma+\Gamma_{\mathrm{ET}} N_{\mathrm{d}}\left(E_{\mathrm{d}}\right) .
$$

In these equations, $N_{\mathrm{d}}$ is the population of excitons at energy $E$, which can be resonantly transferred to larger QDs; $N_{\mathrm{i}}$ is the population of excitons at energy $E$ that cannot be transferred; $N_{\mathrm{a}}$ is the population of excitons at energy $E$ created by transfer of excitons at energy $E_{\mathrm{d}}=E+E_{\mathrm{da}}$ from smaller QDs. $G$ is the exciton generation rate by the excitation, $\Gamma_{\mathrm{ET}}$ and $\Gamma$ are the energy transfer and radiative recombination rates correspondingly. The function $f_{\mathrm{d}}(E)$ determines the probability of excitons to be transferred from initial $\mathrm{DOS}_{1}$. Solving this system of rate equations in the steady state regime we can find the equation for modified density of states

$$
\begin{aligned}
& N_{\mathrm{DOS}_{2}}(E)=N_{\mathrm{DOS}_{1}}(E)-K_{\mathrm{ET}}\left(N_{\mathrm{DOS}_{1}}(E) f_{\mathrm{d}}(E)\right. \\
& \left.-N_{\mathrm{DOS}_{1}}\left(E_{\mathrm{d}}\right) f_{\mathrm{d}}\left(E_{\mathrm{d}}\right)\right) .
\end{aligned}
$$

Here $K_{\mathrm{ET}}=\frac{\Gamma_{\mathrm{ET}}}{\Gamma_{\mathrm{E} T}+\Gamma}$ is the energy transfer efficiency estimated to be close to unity for the parameters of our system. Applying expression (4) to the studied ensemble of QDs, we have derived the modified $\mathrm{DOS}_{2}$ (dashed line in Fig. 2), whose maximum coincides with the PL peak. The function $f_{\mathrm{d}}(\mathrm{E})$ used for calculation of $\mathrm{DOS}_{2}$ spectrum has Gaussian form with the maximum at $2.7 \mathrm{eV}$.

\section{Discussion}

The results of our modeling indicate the possibility of the energy transfer via dipole-dipole interaction between ground state exciton in the small QDs and exciton in one of the excited states in the larger QDs. The Förster energy transfer is not the only mechanism that can occur in arrays of the CdSe/ZnSe QDs. Another possible mechanism of energy transfer could be the diffusion of excitons between QDs through wetting layer [16, 17]. However, we have the following reasons to suggest that in the samples under investigation the Förster energy transfer can dominate. First, additional calculations of higher energy states, performed by direct numerical diagonalization, have shown that the excited levels of needed symmetry do exist in the large QDs. Other necessary prerequisite for realization of such a mechanism is the short distance between the dots, since the efficiency of the resonant energy transfer should depend on the inverse sixth power of the distance between the dots. The characteristic Förster distance $R_{0}$ corresponding to a $50 \%$ transfer efficiency equals $1 \div 10 \mathrm{~nm}$ in different fluorescent systems [18]. In our TEM images, the distance between nearest QDs in the array is usually less than $10 \mathrm{~nm}$. Some deviation at the higher energy tail in the spectrum of $\mathrm{DOS}_{2}$ may be relevant to the simplicity of our straightforward model, when a lot of factors were not taken into account. Actually, not the whole subsystem of the small QDs participates in the energy transfer process. The set of excited levels in the large QDs operates as a band filter, which selects the resonant states in the small QD subsystem. The selected set of the levels can likely provide the higher-energy peaks in the PLE spectra, which have the width comparable with the width of the PL line. 


\section{Conclusions}

Arrays of CdZnSe QDs grown recently possess the bimodal QD size distribution and high dispersion of the QD sizes, which contradicts their very narrow width of photoluminescence lines. Modeling of the density of ground excitonic states in these ensembles gives much broader emission band, whose peak is shifted towards higher energy with respect to the PL line. The results of our modeling, intended to exclude this inconsistency, are suggesting of effective energy transfer in the dense array of epitaxial II-VI QDs. However, this suggestion needs experimental justification, which may be done by PL spectroscopy studies with high temporal resolution. The comparative studies of the arrays with different QD densities could be also recommended.

\section{Acknowledgments}

This work was supported by Russian Science Foundation (Project \#14-22-00107).

\section{References}

[1] S.V. Ivanov, A.A. Toropov, T.V. Shubina, S.V. Sorokin, A.V. Lebedev, I.V. Sedova, P.S. Kop'ev, G.R. Pozina, J.P. Bergman, B. Monemar, J. Appl. Phys. 83, 3168 (1998).

[2] A. Klochikhin, A. Reznitsky, B. Dal Don, H. Priller, H. Kalt, C. Klingshirn, S. Permogorov, S. Ivanov, Phys. Rev. B 69, 085308 (2004).

[3] N. Peranio, A. Rosenauer, D. Gerthsen, S.V. Sorokin, I.V. Sedova, S.V. Ivanov, Phys. Rev. B 61, 16015 (2000).

[4] D. Litvinov, A. Rosenauer, D. Gerthsen, N.N. Ledentsov, Phys. Rev. B 61, 16819 (2000).

[5] D. Litvinov, M. Schowalter, A. Rosenauer, B. Daniel, J. Fallert, W. Löffler, H. Kalt, M. Hetterich, Phys. Status Solidi A 205, 2892 (2008).
[6] S.V. Ivanov, S.V. Sorokin, I.V. Sedova, in: Molecular Beam Epitaxy, Elsevier, Waltham 2013, p. 611.

[7] S.V. Sorokin, S.V. Gronin, I.V. Sedova, M.V. Rakhlin, M.V. Baidakova, P.S. Kop'ev, A.G. Vainilovich, E.V. Lutsenko, G.P. Yablonskii, N.A. Gamov, E.V. Zhdanova, M.M. Zverev, S.S. Ruvimov, S.V. Ivanov, Semiconductors 49, 331 (2015).

[8] A. Tribu, G. Sallen, T. Aichele, R. André, J-P. Poizat, C. Bougerol, S. Tatarenko, K. Kheng, Nano Lett. 8, 4326 (2008).

[9] O. Fedorych, C. Kruse, A. Ruban, D. Hommel, G. Bacher, T. Kümmell, Appl. Phys. Lett. 100, 061114 (2012).

[10] R.N. Kyutt, A.A. Toropov, S.V. Sorokin, T.V. Shubina, S.V. Ivanov, M. Karlsteen, M. Willander, Appl. Phys. Lett. 75, 373 (1999).

[11] K. Kruse, M. Gartner, A. Gust, D. Hommel, Appl. Phys. Lett. 90, 221102 (2007).

[12] S. Adachi, Properties of Semiconductor Alloys: Group-IV, III-V and II-VI Semiconductors, Wiley, Chippenham 2009.

[13] A.A. Golovatenko, M.A. Semina, A.V. Rodina, in: Proc. XIX Symp. "Nanophysics \& Nanoelectronics", Nizhny Novgorod (Russia), Eds.: M.L. Timoshenko, V.V. Sheina, Nizhni Novgorod University Press (NNUP), Nizhni Novgorod 2015, p. 461.

[14] B.L. Gel'mont, M.I. D'yakonov, Fiz. Techn. Poluprovodn. 5, 2191 (1971).

[15] T. Förster, Ann. Phys. (Germany) 437, 55 (1948).

[16] F. Tinjod, S. Moehl, K. Kheng, B. Gilles, H. Mariette, J. Appl. Phys. 95, 102 (2004).

[17] S. Mackowski, G. Prechtl, W. Heiss, F.V. Kyrychenko, G. Karczewski, J. Kossut, Phys. Rev. B 69, 205325 (2004).

[18] J.R. Lakowicz, Principles of Fluorescence Spectroscopy, Springer, New York 2006. 